\title{
The Nano-mechanical Characteristic Experimentation Analysis of Aerial Film Material
}

\author{
Jun Xie ${ }^{1, a}$, Yingnan $\mathrm{Liu}^{2, \mathrm{~b}}$, Kai Xie $\mathrm{Xic}^{3, \mathrm{c}}$, Wei Han ${ }^{1, \mathrm{~d}}$ and Zhonglin $\mathrm{Xu}^{1, \mathrm{e}}$ \\ ${ }^{1}$ Aerospace Intelligence Department, Air Force Aerial University, Changchun, Jilin, 130021, China \\ ${ }^{2}$ ChangChun Automobile Industry Institute, Changchun, Jilin, 130013, China \\ ${ }^{3}$ The army of 95806, Beijing, 100076, China \\ axiejun1981@gmail.com, ${ }^{b}$ liuyingnan2005@163.com, ’xiekai2015@163.com, dhwa3@163.com, \\ exu_zhonglin@sina.com
}

\begin{abstract}
Keywords: aerial film, photographic material, nano-mechanical, characteristic analysis
Abstract. Nano measurement technology is an important means of nano-mechanical properties of thin materials research. Aerial film has more excellent physical and mechanical properties compared to common film. Nano indentation continuous stiffness testing method is applied in the paper, the nano-mechanical properties test of aerial film material analysis is carried out, and the nano-mechanical properties of the material are obtained without emulsion layer and the base material separation, the load-displacement curve was continuous recorded, the calculation values of aerial film material nano-mechanical properties are acquired, and the subsequent research theoretical foundation of the correlation of film image quality and its nano mechanical properties are provided.
\end{abstract}

\section{Introduction}

Aerial camera is an important implement tool in imaging reconnaissance, the ground targets electromagnetic wave radiation or reflections are collected through the optical system, it is focusing on the focal plane, and the energy were provided for recording medium. The medium can be film or photoelectric storage. The digital imaging and digital image material have been developed rapidly since the 1990s, it has the characteristics of imaging convenient storage, real-time transmission, and it has brought the huge impact to the chemical photosensitive material, but the photographic material is still the most mature technology and with most application advantages with the low cost, high resolution, high cost performance and high sensitivity. At the same time because of the excellent properties of biological material and the reflection of the novel material design thought, the frontier and hot issue has become the research field. There are many scientific researches on nano-mechanical properties of biological materials, and it has guiding significance to the research results, and the theoretical guidance was provided for nano-mechanical properties of aerial film [1-5].

\section{The imaging mechanism of film material and its structure}

The film is a kind of photographic material which can accept information, then after the chemical or physical process, a fixed image can be obtained. The nitric acid fiber, acetate fiber or polyester was taken as the base material, on the base coated by gelatin and suspension of photosensitive material made of silver halide particles in emulsion. When screening, the light through the camera lens illuminate on the film emulsion layer, the light reaches the silver halide crystal mass formed by the coalescence and silver halide crystals, and different intensity of illumination can make the film emulsion layer micro field have different amount of crystal structure changes and mutual coalescence, film exposure, latent image, developing the film for operation, the structure change of silver halide crystals into black metallic silver particles get nexus of contracts, produce images; The without photosensitive part on film, the crystals without structure change is washing out by fixative, present light grey or transparent; The result is negative as the dark parts are more exposed, and the bright part is less exposure, the transparent part is not under the light irradiation, it is the black and white film process. There are three emulsion layers in the color film, respectively in the emulsion layer can 
produce dye containing different color coupler. The emulsion layers are colorless, when it was developing, the color development developer oxide coupling to be colored dyes. Compared to the common film, the air film have more excellent physical and mechanical properties such as excellent resistance to impact, high tensile strength, bending strength, compression strength, small creep, size stable, and the relationship between imaging quality and its characteristic parameters depends on the material structure and properties of the film itself, so the air film nano-mechanics experimentation analysis has important practical significance [3].

\section{Nano-mechanical measuring theory}

Nano-mechanical measuring technology is nano indentation technique combined with the continuous stiffness measurement technology, and the mechanical properties of thin film materials were access obtained without separation membrane and the basal material, the characteristic was very well which enable it to analysis the mechanical properties of thin film that the traditional mechanical testing technology was not easy to achieve. The hardness is a mechanical property; it is the integrated performance of chemistry properties, physical properties and structure of the material. According to the different materials, the hardness performance is different. As the performance of the materials, a variety of mechanical properties such as hardness and strength of material, wear resistance, elasticity, plasticity, toughness, etc all have inseparable relationship between physical quantities. Due to the characteristics of intuitive and convenient of hardness measurement, it has become an important research method [2].

The traditional hardness measurement method is to press the specific shape and size of the pressure head into material surface under a certain load, measured after unloading the projection area of the indentation, hardness is obtained by computational load and projection area of the ratio. But this method cannot be used for measuring indentation depth of nano-scale. In recent years, nano hardness measurement using nanometer technology indentation, generally due to the nano indentation technology in small size range, the mechanical properties of the test material, in addition to plastic properties can also reflect the elastic properties of material, can obtain the nano-scale material hardness, has been widely used in material surface engineering, biological and medical materials, thin films and viscoelastic polymer and plastic materials such as testing.

In order to calculate the hardness and elastic modulus values of the material from the load-displacement curve, the elastic contact stiffness and contact area must be known accurately firstly. According to the method of Oliver-Pharr [1,2], the load-displacement fitting relationship of the $60 \%-80 \%$ of the unloading curve top section was shown in Eq. 1 :

$$
F=B\left(h-h_{f}\right)^{m}
$$

$\mathrm{B}$ and $\mathrm{m}$ are obtained by fitting parameters calculation, hf is the displacement after complete discharge. Elastic contact stiffness can be calculated according to the Eq. 2:

$$
S=\left(\frac{d F}{d h}\right)_{h=h_{\max }}=B m\left(h_{\max }-h_{f}\right)^{m-1}
$$

For elastic contraction, the contact depth (hc) can be calculated by Eq. 3:

$$
h_{c}=h-\varepsilon \frac{F}{S} h_{c}=h-\varepsilon \frac{F}{S}
$$

$\varepsilon$ is a constant which related to the pressure head shape, for a Berkovich triangular pyramid head, $\varepsilon=0.75$. For an ideal Berkovich pressure head, contact area can be obtained from Eq. 4 :

$$
A=24.56 h_{c}^{2}
$$

If the material sample is isotropic, its geometry size is greater than the size of the indentation, and the material surface level off, the hardness and Er's modulus calculation formula is as follows:

$$
H=\frac{F}{A}
$$




$$
E_{r}=\frac{\sqrt{\pi}}{2 \beta} \frac{S}{\sqrt{A}}
$$

$\mathrm{F}$ is the indentation depth of real-time load, $\mathrm{A}$ is the area of contact surface. The nano indentation hardness is a measure of the material of contact load capacity according to the formula. Er can be used to explain the elastic deformation of the pressure head and the sample, $\beta$ is a constant that related to the pressure head. For Berkovich pressure head, $\beta=2.034$. The elastic modulus $E$ can be calculated according to the $\mathrm{E}_{\mathrm{r}}$ :

$$
\frac{1}{E_{r}}=\frac{1-v^{2}}{E}+\frac{1-v_{i}^{2}}{E_{i}}
$$

$E$ and $v$ are elastic modulus and poisson's ratio of the tested material; $E_{i}$ and $v_{i}$ are the elastic modulus and poisson's ratio of the pressure head respectively. If the value of $v$ are known, its elastic modulus E's value can be calculated [2].

\section{Experimentation method}

Nano indentation test instrument is American hysitron Triboindenter nano-mechanics test system, as shown in Fig. 1.
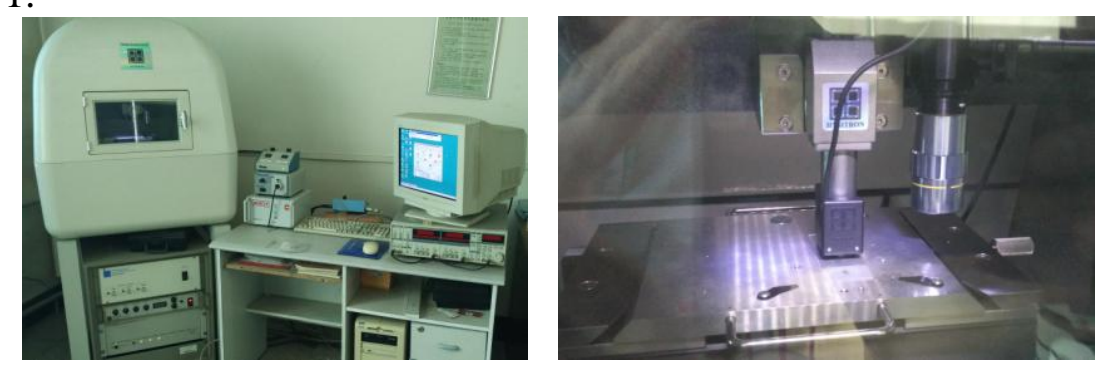

Fig.1 hysitron Triboindenter nano-mechanics test system

The experiment materials sample preparation. To facilitate the comparison and analysis, three kinds of air visible light film were chose as the experiment sample, they are lucky 1023, TYPE C1021 and TYPE C1025. They are marked as sample 1, sample 2 and sample 3 respectively. Sample 1 is unused product, but due to optical environment and the limitation of experimental conditions, the sample 1 was exposed, there were less influence to the experiment result due to the structure characteristic of the film itself, so the effect is neglected. The sample 2 and sample 3 have been shoot exposure. Three samples were adhibit on the glass slide with $\mathrm{AB}$ adhesive and placed on the slide table respectively; ensure that the surface parts under test and the needle tip of nano mechanics test system in a vertical state, the environment conditions of normal temperature and humidity.

The experimental process. The test of elastic modulus and nano hardness were taken in different loading rate and holding time conditions. It was determined that the best loading rate was $50 \mu \mathrm{N} / \mathrm{s}$, the holding time was $10 \mathrm{~s}$, then the credibility and reliability of test results were guaranteed. Six times indentation test were taken on the $0.01 \mathrm{~mm} \times 0.01 \mathrm{~mm}$ area, the average value was taken as the nano indentation performance. The load-displacement curves of three samples are shown in Fig. 2.
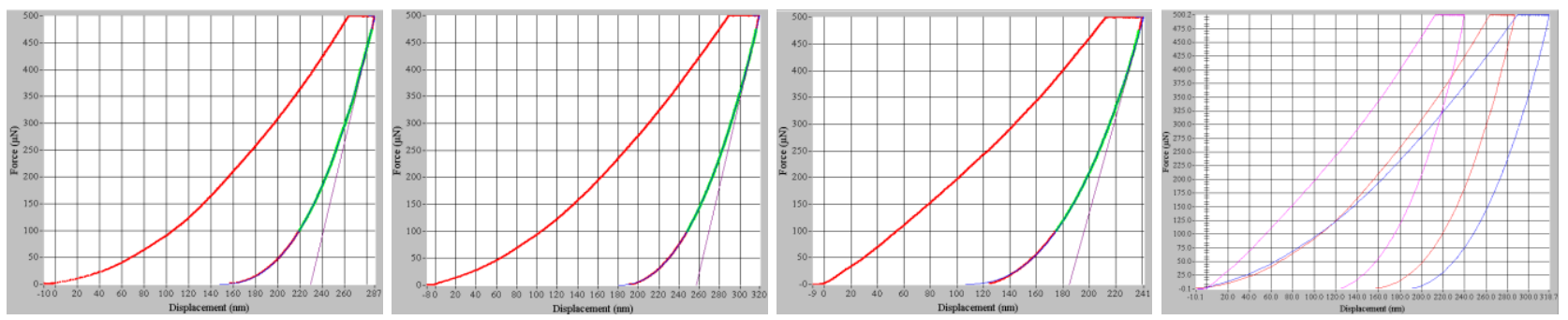

Fig.2 The load-displacement curves of three samples 


\section{The result analysis}

In the process of loading force, firstly the elastic deformation was occurred, along with the increase of the loading force, plastic deformation was gradually occurred, the loading curve was nonlinear. The deformation recovery process of the tested object was reflected by unloading curve, it is an elastic recovery process. The curves contained the information of hardness and elastic modulus. It can be seen from the load-displacement curve as shown in figure 2 that during the initial loading phase, displacement increases with the increase of load, and forming indentation; And in the holding stage, the creep phenomenon can be seen; Then the displacement were fell with the decrease of the load. When load decreases to zero, however, displacement did not return to zero, it was shown that the plastic deformation has been formed.

The nano hardness tester could induct real-time depth change quantitatively. It could obtain the performance parameters of material elastic modulus $\mathrm{E}$, hardness $\mathrm{H}$, stiffness $\mathrm{S}$, maximum depth $\mathrm{D}$, contact depth $\mathrm{h}$ through the continuous recording of the load-displacement curve of loading and unloading as shown in Table 1.It can be found that the mechanical parameters of the three samples are different. The greater the hardness, the smaller the maximum depth and the contact depth, and due to the correlation of the hardness and stiffness, the greater the hardness, the greater the stiffness, and the elastic modulus is greater, they are directly proportional relationship.

Table 1 The Nano-mechanical measuring results

\begin{tabular}{|c|c|c|c|c|c|}
\hline Sample number & E $($ GPa $)$ & H $(\mathbf{G P a})$ & D $(\mathbf{n m})$ & h $(\mathbf{n m})$ & S $(\boldsymbol{\mu n} / \mathbf{n m})$ \\
\hline 1 & 6.45 & $\mathbf{0 . 3 5}$ & 286.77 & 243.79 & 8.66 \\
\hline 2 & 5.41 & 0.29 & $\mathbf{3 1 8 . 7 4}$ & $\mathbf{2 7 2 . 7 6}$ & $\mathbf{8 . 0 2}$ \\
\hline 3 & 7.877 & $\mathbf{0 . 5 0 8}$ & $\mathbf{2 3 9 . 9 8}$ & 198.88 & 8.86 \\
\hline
\end{tabular}

\section{Conclusions}

Although digital transmission technology has become the mainstream in today's aerial filming, but the film is still one of the most mature and application advantages of imaging materials for its high resolution and high cost performance characteristics. The nano mechanical properties of aerial film materials were studied in the paper by using nano mechanics test system.

The nano mechanical properties and load-displacement curve were obtained without separating emulsion layer and the base material, a variety of film materials mechanical performance parameters were acquired through the continuous recording of the loading and unloading curve, the nano mechanics analysis method is introduced into the analysis and research of the air film, theoretical and experimental basis were provided for subsequent nano mechanical properties of film materials and its relationship between the properties and its imaging quality.

\section{Acknowledgements}

This work was financially supported by the Jilin Province Education Department "twelve-five" Science and Technology Research Project ( [2015] no. 449).

\section{References}

[1] J.Y. Sun, Ph.D. Thesis of Jilin University. (2005)

[2] Y.R. Zhao, Ph.D. Thesis of Jilin University. (2007)

[3] J.H. Bao: The imaging system of Optical reconnaissance equipment (Blue Sky Publications, Beijing 2008).

[4] C.L. Bai: Chin. Sci. Bull. Vol. 46 (2001), p. 2

[5] Z.K. Zhang, Z.L. Cui: The imaging system of Optical reconnaissance equipment (National Defence Industry Press, Beijing 2000). 